\title{
Receptor activator of NF-KB ligand (RANKL) is expressed in chondroblastoma: possible involvement in osteoclastic giant cell recruitment
}

\author{
L Huang, Y Y Cheng, L T C Chow, M H Zheng, S M Kumta
}

See end of article for authors' affiliations

.....................

Correspondence to: Professor S M Kumta, Department of Orthopaedics and

Traumatology, The Chinese

University of Hong Kong,

Shatin, NT, Hong Kong

SAR;

sm-kumta@cuhk.edu.hk

Accepted for publication 10 October 2002

\begin{abstract}
Aims: Chondroblastoma is a rare, locally aggressive bone tumour that causes osteolytic destruction at the epiphyseal end of the affected bone. It is possible that tumour cells may stimulate osteoclastogenesis and osteolytic destruction through the production of receptor activator of NF-kB ligand (RANKL), which is a key molecule essential for regulating osteoclast formation and activity. Therefore, the expression of RANKL at both the mRNA and the protein level was investigated in chondroblastoma tumour tissue obtained from patients.

Methods: The expression of RANKL gene transcripts was analysed by the reverse transcriptionpolymerase chain reaction (RT-PCR), and the cellular localisation of RANKL mRNA and protein was demonstrated by means of in situ hybridisation and immunohistochemistry.

Results: RT-PCR analysis indicated that RANKL mRNA was present in all chondroblastoma specimens and normal cancellous bone samples, but not in normal articular cartilage and chondrosarcoma tissues. In contrast, gene transcripts of osteoprotegerin (OPG), the decoy receptor of RANKL, were detected in all types of tissues. The chondroid origin of neoplastic mononuclear cells in chondroblastoma was confirmed by positive S-100 immunohistochemical staining. Both RANKL mRNA and protein were exclusively expressed in these neoplastic mononuclear cells.

Conclusions: These findings suggest that RANKL may be involved in the tumour cell induced recruitment of osteoclast-like cells and consequent osteolytic bone destruction in chondroblastoma.
\end{abstract}

C hondroblastoma is a rare primary bone tumour, which is characterised by massive bone destruction at the epiphydominant components: the neoplastic mononuclear cells (chondroblastic-like cells), multinucleated osteoclast-like giant cells, and chondroid matrix fragments. ${ }^{12}$ Chondroblastoma has been confused with giant cell tumour of bone (GCT) because of the presence of abundant multinuclear giant cells. However, the immunohistochemical demonstration of protein S-100 positivity in the tumour cells of chondroblastoma but not in GCT can help distinguish between these two tumours and is indicative of the chondroid origin of chondroblastoma. ${ }^{3}$ However, multinucleated giant cells in these two types of tumour are identical and are thought to be reactive osteoclasts. A previous study has demonstrated the expression of several osteoclast specific enzymes, including the tartrate resistant acid phosphatase and carbonic anhydrase II, in the multinucleated giant cells of chondroblastoma. ${ }^{5}$ In general, it is thought that the local osteolytic destruction of host bone results from local osteoclast activation. However, the mechanism by which osteoclasts are recruited and activated in chondroblastoma remains unclear.

\footnotetext{
"Our previous work has shown that RANKL is involved in tumour cell induced osteoclastogenesis in GCT, and suggests the possibility that this RANKL-OPG-RANK system also plays an important role in pathological bone destruction in chondroblastoma"
}

The discovery of a novel cytokine system-the tumour necrosis factor family members, receptor activator of NF- $\mathrm{KB}$ ligand (RANKL, also named ODF, OPGL, and TRANCE) ${ }^{6-9}$; its receptor, receptor activator of NF- $\mathrm{KB}(\mathrm{RANK})^{10}$; and its decoy receptor, osteoprotegerin (OPG, also named OCIF $)^{11}{ }^{12}$ - has established a common mechanism by which osteoclast formation and activ- ity is regulated in normal bone. In brief, RANKL, a transmembrane molecule produced by osteoblasts/stromal cells, binds to RANK, which is present on the surface of osteoclast precursors, to stimulate osteoclast precursors to differentiate into osteoclasts. OPG, also produced by osteoblasts/stromal cells, competitively binds to RANKL, which results in the blocking of RANKL and RANK binding, and consequently induces the inhibition of osteoclastogenesis. Our previous work has shown that RANKL is involved in tumour cell induced osteoclastogenesis in $\mathrm{GCT}^{13}$ and suggests the possibility that this RANKL-OPG-RANK system also plays an important role in pathological bone destruction in chondroblastoma. ${ }^{13}$ Therefore, to determine whether RANKL is involved in the tumour cell induced osteoclast recruitment and consequent osteolytic bone destruction in chondroblastoma, we investigated the expression of RANKL at both the mRNA and the protein level in chondroblastoma tissues, using reverse transcription-polymerase chain reaction (RT-PCR), in situ hybridisation (ISH), and immunohistochemistry (IHC) methods.

\section{MATERIALS AND METHODS \\ Materials}

Five chondroblastoma specimens were collected from patients after surgery at the Prince of Wales Hospital (Hong Kong) and the Queen Mary Hospital (Hong Kong), including three

\footnotetext{
Abbreviations: BSA, bovine serum albumin; DIG, digoxigenin; GCT, giant cell tumour of bone; GAPDH, glyceraldehyde-3-phosphate dehydrogenase; Ig, immunoglobulin; IHC, immunohistochemistry; ISH, in situ hybridisation; MMLV, Moloney murine leukaemia virus; OPG, osteoprotegerin; PBS, phosphate buffered saline; RANKL, receptor activator of NF-KB ligand; RT-PCR, reverse transcription-polymerase chain reaction; SCC, saline sodium citrate
} 
Table 1 Chondroblastoma case report

\begin{tabular}{lllll}
\hline \multicolumn{2}{l}{ Case number Sex } & Age (years) & Site & Stage \\
\hline 1 & Male & 19 & Distal femur & III \\
2 & Male & 20 & Proximal tibia & II \\
3 & Female & 13 & Distal femur & III \\
4 & Female & 10 & Distal femur & III \\
5 & Female & 13 & Distal femur & II \\
\hline
\end{tabular}

samples that were freshly frozen down in liquid nitrogen and then stored at $-80^{\circ} \mathrm{C}$ for RNA extraction. Three chondrosarcoma specimens and one normal cancellous bone and one normal articular cartilage were also freshly collected from patients after surgery at the Prince of Wales Hospital. TRIzol ${ }^{\circledR}$ reagent for RNA isolation was purchased from Gibco Life Technologies (Hong Kong), Moloney murine leukaemia virus (MMLV) reverse transcriptase and Taq DNA polymerase from Promega (Madison, USA), and DIG (digoxigenin) RNA labelling kit and DIG nucleic acid detection kit from Roche Diagnostics (Mannheim, Germany). Mouse antihuman TRANCE monoclonal antibody was purchased from R\&D Systems Inc (Minneapolis, Minnesota, USA) and rabbit anti-S100 immunoglobulin (Ig) fraction was from Dako (Sydney, Australia). The Strept ABComplex/HRP antimouse/rabbit kit and $\mathrm{DAB}$ substrate chromogen system were purchased from Dako (Carpinteria, California, USA). All other chemicals used were of the highest grade available.

\section{RNA extraction and RT-PCR}

Total RNA was isolated from chondroblastoma, chondrosarcoma, and normal bone and cartilage tissues by the use of TRIzol reagent according to the manufacturer's instructions. Single stranded cDNA was then prepared from the total RNA using $100 \mathrm{U}$ of M-MLV reverse transcriptase for each reaction with an oligo-dT primer (Promega). Aliquots of $1 \mu \mathrm{l}$ of each cDNA were subjected to PCR using human RANKL, OPG, and glyceraldehyde-3-phosphate dehydrogenase (GAPDH) specific primers described previously. ${ }^{13}$ PCR was performed in a DNA thermal cycler (model PTC-200; MJ Research, Massachusetts, USA). The PCR products were electrophoresed on a $1.5 \%$ agarose gel in the presence of ethidium bromide, and absorbance was measured by a densitometer.

\section{In situ hybridisation}

DIG labelled RANKL antisense riboprobes were prepared as described previously. ${ }^{13}$ In situ hybridisation was performed as described, ${ }^{13}$ with minor modifications. In brief, paraffin wax embedded chondroblastoma tissue sections, which were collected on slides pretreated with 3-aminoprophyltriethoxysilane, were dewaxed with xylene, and then dehydrated in ethanol with sequential concentrations from $100 \%$ to $70 \%$ before rinsing in DEPC treated water. Tissues were then treated with $0.2 \%$ Triton X-100/phosphate buffered saline (PBS) for five minutes, followed by digestion with $2 \mu \mathrm{g} / \mathrm{ml}$ proteinase $\mathrm{K}$ in $0.1 \mathrm{M}$ Tris buffer ( $\mathrm{pH} 8.0$ ) with $50 \mathrm{mM}$ EDTA for $20-30$ minutes at $37^{\circ} \mathrm{C}$. Digestion was stopped with $0.1 \%$ glycine/PBS for two minutes. The tissues were then fixed in $4 \%$ paraformaldehyde/PBS for 15 minutes, followed by DNase treatment (RNase free, $1 \mathrm{U} / \mathrm{ml}$ ) for 30 minutes at $37^{\circ} \mathrm{C}$ to ensure specificity of mRNA hybridisation. Slides were washed in PBS between each treatment and all procedures were carried out at room temperature unless indicated otherwise. After prehybridisation in solution containing 50\% deionised formamide in $5 \times$ saline sodium citrate (SSC) and $100 \mu \mathrm{g} / \mathrm{ml}$ denatured salmon sperm DNA at $37^{\circ} \mathrm{C}$ for one hour, the sections were hybridised overnight at $37^{\circ} \mathrm{C}$ in a humidified chamber with hybridisation solution (50\% deionised formamide and $10 \%$ dextran sulfate in $5 \times$ SSC) containing DIG labelled RANKL riboprobe at a concentration of $0.5 \mathrm{ng} / \mathrm{ml}$. Slides were protected with coverslips during the hybridisation period. After hybridisation, the sections were washed with $2 \times$ SSC twice, $1 \times$ SSC twice, and finally $0.1 \times$ SSC twice at $37^{\circ} \mathrm{C}$ for 15 minutes each. Detection of hybridised probes was then performed by enzyme linked immunoassay with alkaline phosphate conjugated anti-DIG antibody and nitroblue tetrazolium chloride/5-bromo-4-chloro3-indolyl-phosphate colour substrate using the DIG nucleic acid detection kit. In each case, tissues treated with RNase $(100 \mu \mathrm{g} / \mathrm{ml})$ before hybridisation were used as negative controls. Slides were assessed by light microscopy and image processing was performed using MetaMorph (version 4.5 ).

\section{Immunohistochemistry}

Mouse antihuman TRANCE monoclonal antibody (R\&D Systems) and rabbit anti-S100 Ig fraction (Dako, Sydney, Australia) were used, respectively, to assess the expression of the RANKL protein and the S-100 protein in chondroblastoma paraffin wax embedded sections. Briefly, paraffin wax embedded tissue sections were dewaxed with xylene and then dehydrated in sequential diluted ethanol before rinsing in PBS. Endogenous peroxidase activity was blocked with 1\% hydrogen peroxide in PBS for 20 minutes at room temperature. Sections were then microwave treated for 10 minutes in

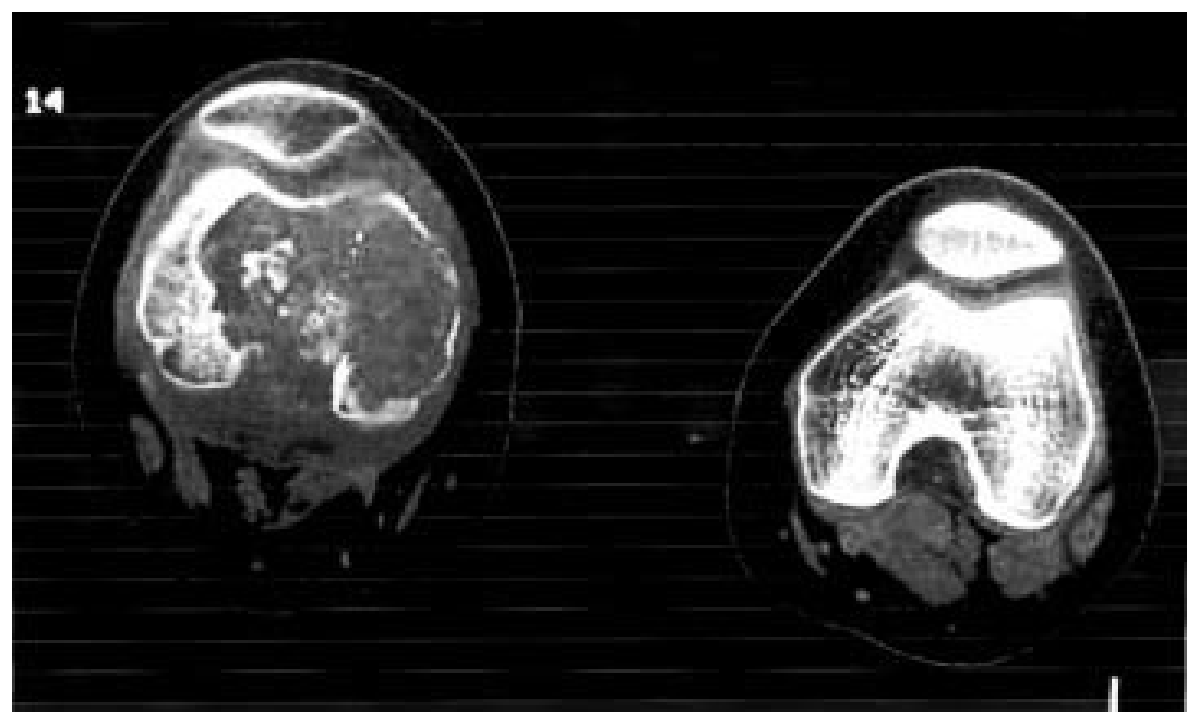

Figure 1 Chondroblastoma affecting medial femoral condyle. Computed tomography scan shows a large expansile lesion affecting the medial femoral condyle and extending into the lateral. Cortical thinning expansion and disruption are noted. 

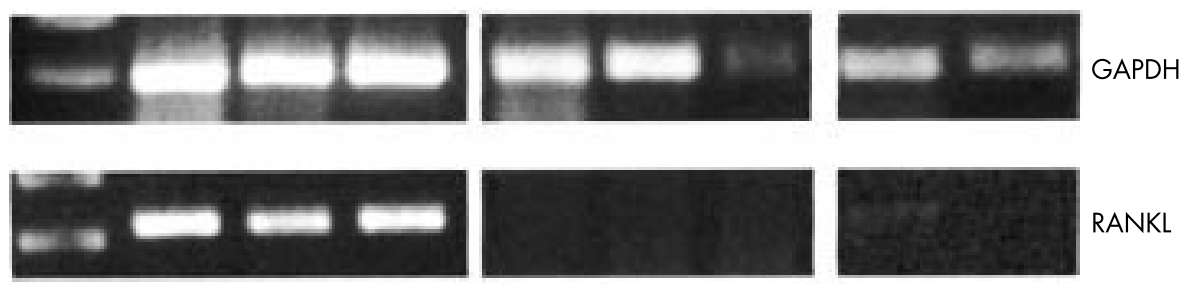

RANKL
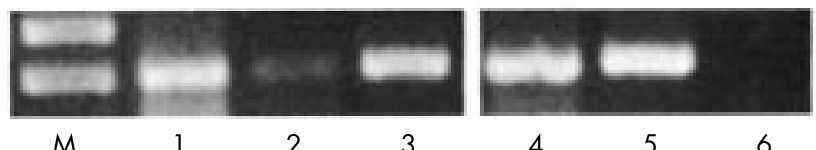

4

5

6

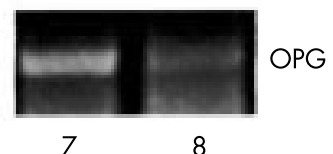

Figure 2 Gene expression of receptor activator of NF-kB ligand (RANKL) in chondroblastoma by reverse transcription-polymerase chain reaction. Lane M, 100 bp DNA ladder; lanes 1-3, chondroblastoma; lanes 4-6, chondrosarcoma; lane 7, normal cancellous bone; lane 8, normal cartilage. The sizes of the glyceraldehyde-3-phosphate dehydrogenase (GAPDH), RANKL, and osteoprotegerin (OPG) PCR products were 206 bp, 486 bp, and 324 bp, respectively.

$0.01 \mathrm{M}$ citrate buffer ( $\mathrm{pH} 6.0$ ) to unmask the antigens. Before being incubated with mouse antihuman TRANCE antibody or rabbit anti-S100 Ig fraction (dilution, 1/100 in 1\% bovine serum albumin (BSA) in PBS) at $4^{\circ} \mathrm{C}$ overnight, sections were incubated with $5 \%$ normal goat serum in $1 \%$ BSA in PBS for 30 minutes to block non-specific IgG binding. A biotinylated goat antirabbit/mouse IgG (dilution, 1/200) was used for further incubation, and a strepavidin-biotin complex system with diaminobenzidene as chromogen was used for colour development. Sections were finally counterstained with haematoxylin and examined under a light microscope.

\section{RESULTS}

\section{Clinical findings}

We studied five chondroblastoma specimens from patients aged from 10-20 years. Histological confirmation of the diagnosis in each case was based on the review of routinely prepared paraffin wax embedded tissue sections in conjunction with knowledge of the clinical and radiological findings. As shown in table 1, all five cases were either stage II or stage III chondroblastoma, with aggressive osteolytic lesions on radiological examination (fig 1 ).

\section{Gene expression of RANKL in chondroblastoma}

To determine whether RANKL gene transcripts were expressed in chondroblastoma, RNA isolated from snap frozen samples of three patients (numbers 1,2, and 3 in table 1) were subjected to RT-PCR analysis. A total of 25 cycles were used to amplify the GAPDH gene transcript, which was used as an internal control, and 30 cycles for RANKL and OPG gene expression. As shown in fig 2, all three cases of chondroblastoma expressed both RANKL and OPG gene transcripts ( fig 2, lanes 1-3). In contrast, RANKL mRNA was undetectable in three stage II chondrosarcomas (fig 2, lanes 4-6), even when the number of PCR amplification cycles was increased to 40 (data not shown). OPG gene expression was variable in chondrosarcoma tissues, being detectable in two of three patients (fig 2, lanes 4-6). Both RANKL and OPG gene transcripts were undetectable in normal cancellous bone and cartilage when 30 amplification cycles were used (data not shown); however, RANKL mRNA was present in normal bone tissues and OPG mRNA was present in both normal bone and cartilage when the number of amplification cycles was increased to 40 (fig 2, lanes 7-8).

\section{Cellular localisation of RANKL in chondroblastoma}

To examine the cellular localisation of RANKL in various cell components of chondroblastoma, ISH using DIG labelled human RANKL riboprobe and IHC using mouse antihuman
TRANCE monoclonal antibody were performed in all five cases of chondroblastoma. Figure 3 shows representative images for ISH and IHC. RANKL mRNA was exclusively expressed in the cytoplasm of the neoplastic mononuclear cells of chondroblastoma, but not in the osteoclast-like giant cells (fig 3B, C). IHC results were similar to those with ISH, showing strongly positive immunoreactivity for RANKL protein in the neoplastic mononuclear cells (fig 3E). It is noteworthy that the RANKL positive neoplastic mononuclear cells were also positive for $\mathrm{S}$ - 100 protein (fig $3 \mathrm{G}, \mathrm{H}$ ), indicating the chondroid origin of these tumour cells.

\section{DISCUSSION}

Chondroblastoma has been described as a benign bone neoplasm, which usually affects the epiphyseal end of the bone. Massive osteolytic bone destruction at the epiphyseal end may eventually result in joint destruction and cause significant functional consequences. There is no evidence that tumour cells themselves are capable of bone resorption, instead, osteoclast-like giant cells, the reactive cell components in chondroblastoma, are responsible for the bone resorbing activity. ${ }^{515}$ We and others have shown previously that RANKL is present in the tumour cells of GCT, ${ }^{13}{ }^{16}$ another osteolytic bone neoplasm with the presence of abundant multinucleated osteoclast-like cells in the lesions. Moreover, it was suggested that the ratio of RANKL : OPG expression in tumour cells might contribute to the degree of osteoclastogenesis and bone resorption in GCT. ${ }^{13}{ }^{17}$ Here, for the first time, we report that both RANKL mRNA and protein were also present in the tumour cells of chondroblastoma. Given the small number of cases in our study, the potential prognostic value of RANKL expression in this tumour is still uncertain because no obvious correlation between the degree of RANKL expression and the clinical stage of this tumour was seen.

"Adjuvant drugs that could counteract the effect of RANKL might be useful for reducing the osteolytic potential of this tumour"

It is noteworthy that RANKL mRNA was not present in three grade II chondrosarcomas as measured by RT-PCR analysis. Chondrosarcoma is different from chondroblastoma and GCT, in that it is a malignant lesion that usually produces a chondroid matrix. ${ }^{1}$ Osteoclastogenesis is not often seen in chondrosarcoma, and the calcification and/or ossification are often seen within the depths of the lesion. ${ }^{1}$ It has previously been reported that two cysteine proteinases-cathepsin B and cathepsin L-are highly expressed in high grade and recurrent 

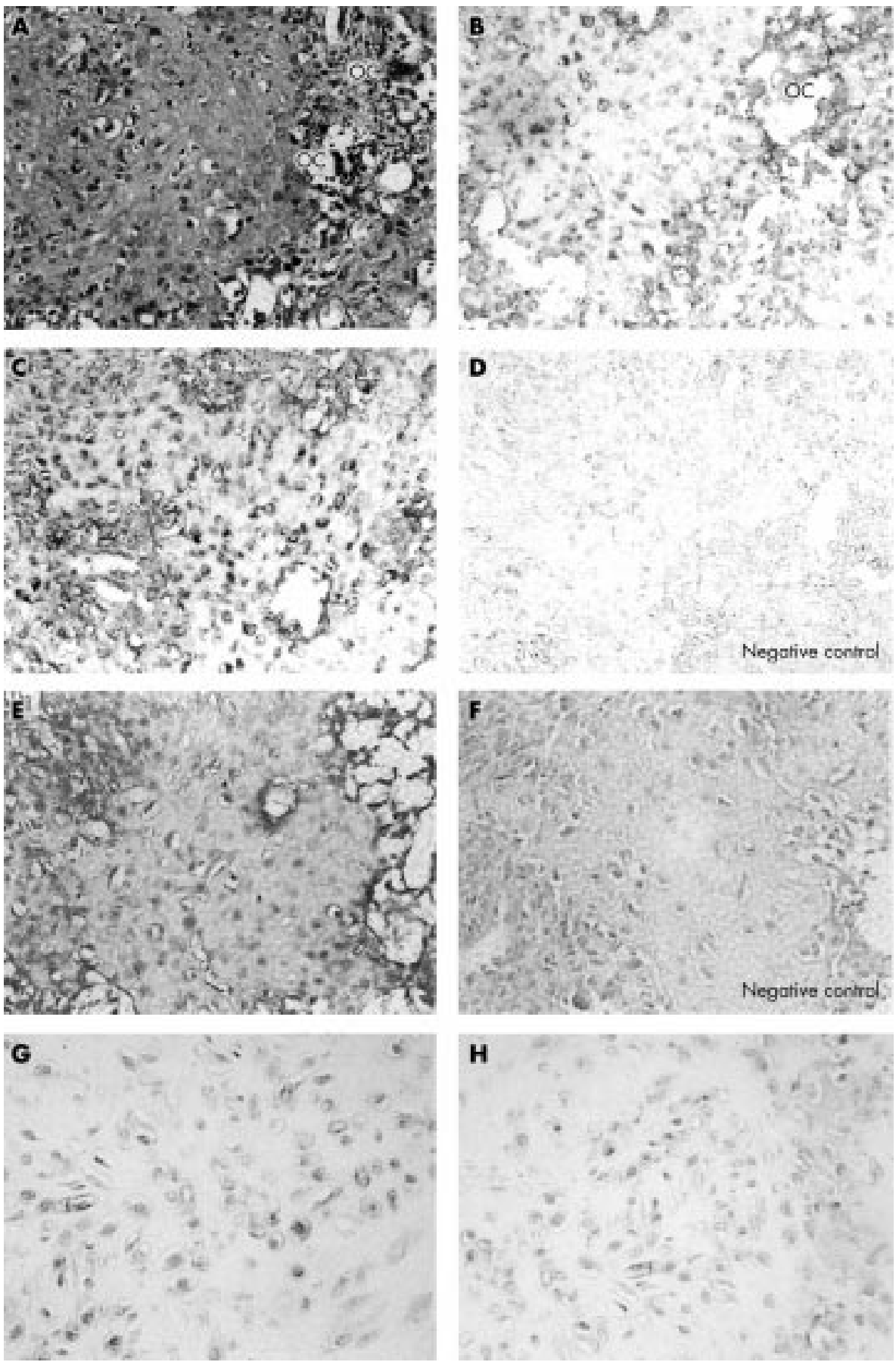

Figure 3 Cellular localisation of RANKL in chondroblastoma by in situ hybridisation (ISH) and immunohistochemistry (IHC). (A) Histological appearance of chondroblastoma by haematoxylin and eosin staining. The mononuclear cells show distinct cytoplasmic boundaries and focal mineralisation is seen between individual tumour cells. Scattered osteoclast-like giant cells (OC) are also present. (B, C) RANKL gene expression is detected in the neoplastic mononuclear cells by ISH. Hybridisation signals are dark blue in colour. No hybridisation signals were seen in osteoclast-like giant cells (OC). (D) The ISH negative control treated with RNase before hybridisation shows no detectable signals for RANKL gene transcripts. (E) RANKL immunoreactivity is detected in the cytoplasm of neoplastic mononuclear cells. Immunoreactivity is seen as a brown colour. (F) The negative control for RANKL IHC shows the specificity of the primary antibody. (G, H) RANKL positive neoplastic mononuclear cells show a positive reaction for S-100 protein. Immunoreactivity is seen as a brown colour.

chondrosarcoma, suggesting the involvement of cathepsins in tumour progression. ${ }^{18}$ The aggressive biological behaviour of chondrosarcoma may be more related to the synthesis of cysteine proteinases and the activation of other proteolytic enzymes than the production of the osteoclast stimulator RANKL.

In summary, the presence of RANKL, the crucial bone resorption regulator, in chondroblastoma tumour cells suggests that tumour cells may use the RANKL-OPG axis to induce osteoclastogenesis, which results in osteolytic bone destruction in this tumour. Thus, adjuvant drugs that could counteract the effect of RANKL might be useful for reducing the osteolytic potential of this tumour.

\section{ACKNOWLEDGEMENTS}

This study was fully supported by a grant of the Research Grants Council of the Hong Kong SAR (project number, CUHK/4142/00M). 


\section{Take home messages}

- RANKL, a crucial bone resorption regulator, was present in chondroblastoma tumour cells but not in chondrosarcomas

- RANKL may be involved in the tumour cell induced recruitment of osteoclast-like cells and the consequent osteolytic bone destruction seen in chondroblastoma

- Adjuvant drugs that could counteract the effect of RANKL might be useful for reducing the osteolytic potential of chondroblastoma

\section{Authors' affiliations}

L Huang, Y Y Cheng, S M Kumta, Department of Orthopaedics and Traumotology, Chinese University of Hong Kong, Shatin, NT, Hong Kong SAR

L T C Chow, Department of Anatomical and Cellular Pathology, Chinese University of Hong Kong

M H Zheng, Department of Orthopaedic Surgery, University of Western Australia, WA 6009, Australia

\section{REFERENCES}

1 Unni KK. Benign chondroblastoma. In: Dahlin's bone tumors: general aspects and data on 11,087 cases, 5th ed. Lippincott-Raven Publishers, 1996:47-58.

2 Fanning CV, Sneige NS, Carrasco CH, et al. Cancer fine needle aspiration cytology of chondroblastoma of bone. Cancer 1990;65: 1847-63

3 Okajima K, Honda I, Kitagawa T. Immunohistochemical distribution of S-100 protein in tumors and tumor-like lesions of bone and cartilage. Cancer 1988;61:792-9.

4 Fukuda T, Saito M, Nakajima T. Imprint cytology of chondroblastoma of bone. A case report. Acta Cytol 1998;42:403-6.

5 Toyosawa S, Ogawa Y, Chang CK, et al. Histochemistry of tartrate-resistant acid phosphatase and carbonic anhydrase isoenzyme II in osteoclast-like giant cells in bone tumours. Virchows Arch A Pathol Anat Histopathol 1991;418:255-61.
6 Anderson DM, Maraskovsky E, Billingsley WL, et al. A homologue of the TNF receptor and its ligand enhance T-cell growth and dendritic-cell function. Nature 1997;390:175-9.

7 Yasuda $\mathbf{H}$, Shima N, Nakagawa N, et al. Osteoclast differentiation factor is a ligand for osteoprotegerin/osteoclastogenesis-inhibitory factor and is identical to TRANCE/RANKL. Proc Natl Acad Sci U S A 1998; 95:3597-602.

8 Lacey DL, Timms E, Tan HL, et al. Osteoprotegerin ligand is a cytokine that regulates osteoclast differentiation and activation. Cell 1998; 93: 165-76.

9 Fuller K, Wong B, Fox S, et al. TRANCE is necessary and sufficient for osteoblast-mediated activation of bone resorption in osteoclasts. J Exp Med 1998;188:997-1001.

10 Hsu H, Lacey DL, Dunstan CR, et al. Tumor necrosis factor receptor family member RANK mediates osteoclast differentiation and activation induced by osteoprotegerin ligand. Proc Natl Acad Sci U S A 1999;96:3540-5

11 Simonet WS, Lacey DL, Dunstan CR, et al. Osteoprotegerin: a nove secreted protein involved in the regulation of bone density. Cell 1997;89:309-19

12 Tsuda E, Goto M, Mochizuki S, et al. Isolation of a novel cytokine from human fibroblasts that specifically inhibits osteoclastogenesis. Biochem Biophys Res Commun 1997;234:137-42.

13 Huang L, Xu J, Wood DJ, et al. Gene expression of osteoprotegerin ligand, osteoprotegerin, and receptor activator of NF-kappaB in giant cell tumor of bone: possible involvement in tumor cell-induced osteoclast-like cell formation. Am J Pathol 2000;156:761-7.

14 Zheng MH, Robbins $\mathrm{P}, \mathrm{Xu}$ J, et al. The histogenesis of giant cell tumour of bone: a model of interaction between neoplastic cells and osteoclasts. Histol Histopathol 2001;16:297-307.

15 Edel G, Ueda Y, Nakanishi J, et al. Chondroblastoma of bone. A clinical, radiological, light and immunohistochemical study. Virchows Arch A Pathol Anat Histopathol 1992;421:355-66.

16 Atkins GJ, Haynes DR, Graves SE, et al. Expression of osteoclast differentiation signals by stromal elements of giant cell tumors. J Bone Miner Res 2000;15:640-9.

17 Atkins GJ, Bouralexis S, Haynes DR, et al. Osteoprotegerin inhibits osteoclast formation and bone resorbing activity in giant cell tumors of bone. Bone 2001;28:370-7.

18 Soderstrom $M$, Ekfors $T$, Bohling $T$, et al. Cysteine proteinases in chondrosarcomas. Matrix Biol 2001;19:717-25. 